\title{
Compton Scattering of Fe K $\alpha$ Lines from Accreting White Dwarfs
}

\author{
Zdenka Kuncic $^{\mathrm{A}, \mathrm{D}}$, Kinwah $W u^{\mathrm{B}}$, and Jason G. Cullen ${ }^{\mathrm{C}}$ \\ A School of Physics, University of Sydney, Sydney NSW 2006, Australia \\ B Mullard Space Science Laboratory, University College London, Holmbury St Mary, RH5 6NT, UK \\ ${ }^{C}$ ADI Ltd, Canberra ACT 2612, Australia \\ D Corresponding author. Email: z.kuncic@physics.usyd.edu.au
}

Received 2004 September 17, accepted 2004 November 12

\begin{abstract}
Compton scattering in the bulk accretion flow of the accretion column in magnetic cataclysmic variables can significantly shift photon energies in the X-ray emission lines resulting from accretion shocks. In particular, Compton recoil can potentially broaden the 6.7 and $6.97 \mathrm{keV} \mathrm{Fe} \mathrm{K} \alpha$ emission lines produced in the post-shock region, and contaminate the fluorescent $6.4 \mathrm{keV}$ neutral $\mathrm{Fe} \mathrm{K} \alpha$ line reflected off the white dwarf surface. We present nonlinear Monte Carlo simulations demonstrating these effects, and we discuss the interpretation of observed $\mathrm{Fe} \mathrm{K} \alpha$ linewidths in magnetic cataclysmic variables in light of these new results. The implications for other accreting compact objects are also discussed.
\end{abstract}

Keywords: accretion — line: profiles — scattering — binaries: close — white dwarfs — X-rays

\section{Introduction}

Magnetic cataclysmic variables (mCVs) are low-mass close binaries with a Roche lobe overfilling red dwarf transferring material to a magnetic white dwarf (see, for example, Cropper 1990; Warner 1995). The two main subclasses of mCVs are the polars (AM Herculis systems) and the intermediate polars (IPs). In a polar, the white dwarf has a sufficiently strong magnetic field $(B \sim 10$ $200 \mathrm{MG}$ ) such that the white dwarf and its companion star are locked into synchronous rotation. The magnetic field also prevents the formation of an accretion disk, causing the material from the red dwarf to be accreted onto the white dwarf directly along its magnetic poles. Although the magnetic field of a white dwarf in an IP is weaker, allowing an accretion disk to form, the inner part of the disk is truncated by the white dwarf magnetic field. In both polars and IPs, the accretion flows onto their white dwarfs are confined by the magnetic field, and accretion columns are formed, channelling the accreting matter to the white dwarf magnetic polar regions.

An accretion shock often develops in the accretion column such that the supersonic accretion flow becomes subsonic in the downstream, post-shock region, before settling onto the white dwarf surface. The shock heats the accreting matter to temperatures

$$
k T_{\mathrm{s}} \approx \frac{3}{8} \frac{G M_{\mathrm{w}} \mu m_{\mathrm{H}}}{\left(R_{\mathrm{W}}+x_{\mathrm{s}}\right)},
$$

where $M_{\mathrm{w}}$ and $R_{\mathrm{W}}$ are the white dwarf mass and radius, $\mu$ is the mean molecular weight, and $x_{\mathrm{s}}$ is the shock height, which is typically $\ll R_{\mathrm{w}}$ (see, however, Cropper et al. 1999 for cases with large shock heights). For white dwarf masses of $0.5-1.0 M_{\odot}$ and typical $\mathrm{mCV}$ parameters, the shock temperature is $k T_{\mathrm{s}} \sim 10-40 \mathrm{keV}\left(\sim 1-4 \times 10^{8} \mathrm{~K}\right)$. The hot plasma in the post-shock region is cooled by emitting bremsstrahlung X-rays and optical/infrared cyclotron radiation (Lamb \& Masters 1979; Wu 2000, and references therein).

At a plasma temperature of $k T \sim 1-50 \mathrm{keV}$, heavy elements such as iron are highly ionised, resulting in an abundant population of $\mathrm{H}$ - and He-like ions (see Fujimoto 1998 and $\mathrm{Wu}$, Cropper, \& Ramsay 2001 for the ionisation structure of post-shock flow in $\mathrm{mCVs}$ ). The $6.97 \mathrm{keV}$ Lyman- $\alpha$ line is emitted due to the $2 \mathrm{p}^{2} \mathrm{P} \rightarrow 1 \mathrm{~s}^{2} \mathrm{~S}$ transition in the H-like Fe Xxvi ions. The $6.70 \mathrm{keV}^{4} \mathrm{He}$ line is emitted from the He-like Fe Xxv ions for the $2 \mathrm{p}^{1} \mathrm{P}_{1} \rightarrow 1 \mathrm{~s}{ }^{1} \mathrm{~S}_{0}$ transition; and the 6.67 and $6.68 \mathrm{keV}{ }^{5} \mathrm{He}$ lines, for the $2 \mathrm{p}{ }^{3} \mathrm{P}_{1} \rightarrow 1 \mathrm{~s}{ }^{1} \mathrm{~S}_{0}$ and $2 \mathrm{p}^{3} \mathrm{P}_{2} \rightarrow 1 \mathrm{~s}{ }^{1} \mathrm{~S}_{0}$ transitions respectively. The $6.64 \mathrm{keV}{ }^{6} \mathrm{He}$ line, due to the $2 \mathrm{p}^{3} \mathrm{~S}_{1} \rightarrow 1 \mathrm{~s}^{1} \mathrm{~S}_{0}$ forbidden transition, is generally suppressed in the shockheated regions of mCVs, because of a high $\left(>10^{14} \mathrm{~cm}^{-3}\right)$ electron number density.

The natural widths of these Fe K $\alpha$ lines are small. Realistically, the lines will be Doppler broadened by the bulk motion of the emitters in the flow, and also by scattering processes. X-ray observations of $\mathrm{mCV}$ s have indeed revealed substantially broadened $\mathrm{Fe} \mathrm{K} \alpha$ emission lines (Hellier, Mukai, \& Osborne 1998; Hellier \& Mukai 2004), though flow-velocity induced Doppler shifts are yet to be verified. The line photons may undergo a large number of resonant scatterings with the ions, but this requires the scattering region to have small velocity gradients (see Terada et al. 2001 for a detailed study of resonant scattering in $\mathrm{mCVs}$ ). Electron (Compton) scattering can shift line photon frequencies significantly and thereby allow the 
photons to escape resonance trapping. It also can cause substantial line broadening.

A previous study (Wu 1999) proposed that cold electrons in the upstream pre-shock bulk flow of mCVs can scatter a significant number of line photons emitted from the shock-heated region, and any $6.4 \mathrm{keV}$ fluorescent Fe line from the white dwarf surface could be contaminated by the down-scattered $6.7 \mathrm{keV}$ He-like Fe line photons. A recent study (Matt 2004) showed that such scattering could be important in that it modifies the X-ray polarisation properties. In this paper, we investigate the effects of electron (Compton) scattering in the accretion column of mCVs by means of numerical simulations. We focus on its effects on the H-like, the He-like, and the neutral $\mathrm{Fe} \mathrm{K} \alpha$ lines. We employ a non-linear Monte Carlo algorithm (Cullen 2001a, 2001b), which improves on other similar codes (Stern et al. 1995; Hua 1997) by taking into account nonuniformity in the velocity, density, and temperature profiles in the scattering region. The paper is organised as follows: in Section 2, we briefly discuss various line broadening mechanisms; in Section 3 we outline the geometry and physical parameters of our model for the line-emitting and Compton scattering regions in mCVs, and we summarise the Monte Carlo method used in our simulations; results are presented and discussed in Section 4; and conclusions are given in Section 5.

\section{Broadening of $\mathrm{Fe} \mathrm{K} \alpha$ Lines from $\mathrm{mCVs}$}

The post-shock region of mCVs is mostly optically thin to bremsstrahlung X-rays, and the coronal approximation is usually applicable for line emissivity calculations (e.g. Fujimoto 1998; Wu et al. 2001). The thermal Doppler widths of the $\mathrm{Fe} \mathrm{K} \alpha \mathrm{H}$-like line are about $5 \mathrm{eV}$ at temperatures $\sim 10 \mathrm{keV}$, and the natural width of the line is about $1 \mathrm{eV}$. The Fe lines can, however, be broadened by the velocity dispersion of the accretion flow in the emission region. The infalling material will hit the accretion shock with speeds $\sim 6000 \mathrm{~km} \mathrm{~s}^{-1}$; if the shock is adiabatic, the post-shock speed will be about four times slower. Thus, the velocity dispersion of emission lines in an orbitalphase averaged spectrum could reach up to $1000 \mathrm{~km} \mathrm{~s}^{-1}$, corresponding to a broadening of about $20 \mathrm{eV}$.

Distortion of the line profiles can also be caused by electron (Compton) scattering. A line photon can gain energy through collisions with hot electrons (i.e. $k T_{\mathrm{e}}>E_{\mathrm{c}}$, where $T_{\mathrm{e}}$ is the electron temperature and $E_{\mathrm{c}}$ the line centre energy), and the fractional energy change per scattering is (Pozdnyakov, Sobol, \& Sunyaev 1977)

$$
\frac{\Delta E}{E} \approx \sqrt{\frac{2 k T_{\mathrm{e}}}{m_{\mathrm{e}} c^{2}}} \approx 0.064\left(\frac{k T_{\mathrm{e}}}{1 \mathrm{keV}}\right)^{1 / 2} .
$$

For cold electrons $\left(k T_{\mathrm{e}} \ll E_{\mathrm{c}}\right)$, the photon loses energy due to recoils. For an He-like Fe line, the energy shift per scattering is

$$
\frac{\Delta E}{E} \approx \frac{E}{m_{\mathrm{e}} c^{2}} \approx 0.014\left(\frac{E}{6.7 \mathrm{keV}}\right) .
$$

Although the energy shift per scattering is small, the Fe line can be broadened significantly after multiple scattering.

For an $\mathrm{mCV}$ with a specific accretion rate of $\sim 1-10 \mathrm{~g} \mathrm{~cm}^{-2} \mathrm{~s}^{-1}$, the thickness of the shock-heated region is $x_{\mathrm{s}} \sim 10^{7} \mathrm{~cm}$, and the electron number density is $n_{\mathrm{e}} \sim 10^{16} \mathrm{~cm}^{-3}$. The corresponding Thompson optical depth is $\tau \sim 0.1$. The mean number of scatterings is $N \approx \max \left(\tau^{2}, \tau\right)$ (Rybicki \& Lightman 1979). Thus, one in every ten line photons would encounter an electron before leaving the post-shock region. The effective Doppler broadening due to Compton scattering by hot electrons in this downstream region is about $5 \%$ of the line-centre energy.

Compton scattering by the cold, upstream electrons in the pre-shock region can, on the other hand, cause much more substantial line broadening. Despite the fact that the electron number density in the upstream flow could be an order of magnitude lower than that in the downstream, post-shock region, the linear size of the accretion column between the accretion shock and the magnetospheric coupling region could be more than two orders of magnitude larger $\left(\sim 10^{9} \mathrm{~cm}\right)$ than the thickness of the shock-heated region. The effective line-of-sight electron scattering optical depth $\tau$ is therefore anisotropic, with values larger than unity at certain viewing angles. We would therefore expect observable Compton recoil signatures in the X-ray emission line spectra of mCVs.

Note that line broadening due to resonance scattering is a factor of $\left(m_{\mathrm{e}} / m_{\mathrm{i}}\right)^{1 / 2}$ smaller than broadening due to Compton scattering. For the $\mathrm{Fe} \mathrm{H}$ - and He-like lines, this factor is $\sim 1 / 320$ (Pozdnyakov et al. 1977).

\section{Modelling Compton Scattering in the Accretion Column}

\subsection{Line Emission and Scattering Regions}

We investigate Compton scattering effects on $\mathrm{Fe} \mathrm{K} \alpha$ lines in $\mathrm{mCV}$ accretion columns using Monte Carlo simulations. We use line centre energies of 6.4, 6.675, and $6.97 \mathrm{keV}$, corresponding to transitions of neutral, He-like, and $\mathrm{H}$-like $\mathrm{Fe}$, respectively. The neutral and Lyman- $\alpha$ transitions are actually doublets, with energies $6.391 / 6.404 \mathrm{keV}$ and $6.952 / 6.973 \mathrm{keV}$ and branching ratio $1: 2$, and the He-like transition has both resonant and intercombination components, and dielectronic satellite lines are also associated with the $\mathrm{H}$-like and He-like lines. These subcomponents remain unresolvable with current X-ray instruments and, in this work, we simply use the approximate line centre energies in order to demonstrate the importance of Compton scattering effects on the line profiles.

In the simulations, the line photons are emitted and scattered in the accretion column, which is assumed to be a cylinder with a circular cross-section. The cyclinder is divided into a geometrically thin, downstream shock-heated region, and a semi-infinite, upstream preshock region. The radius of the accretion cylinder is assumed to be $0.1 R_{\mathrm{w}}$. The line photons are emitted in the 


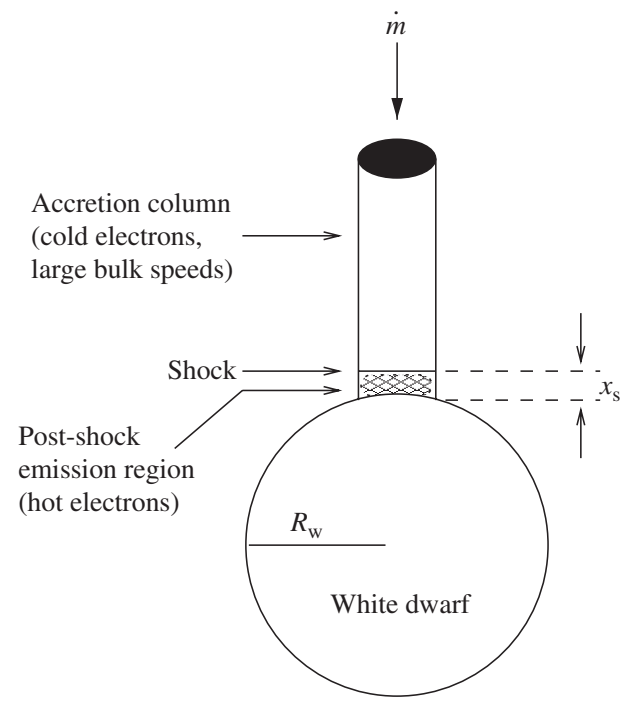

Figure 1 Schematic illustration of the geometry used to model accretion onto a white dwarf, line emission by shock-heated electrons, and Compton scattering by both hot and cold electrons in the accretion column.

shock-heated region, and scattering occurs in both the post- and pre-shock regions. The geometry of the model is shown in Figure 1. We note that the X-ray emission from the post-shock accretion column illuminates the white dwarf atmosphere, which could cause $\mathrm{Fe} \mathrm{K} \alpha$ fluorescence and additional Compton recoil effects. Such effects can be modelled using a Monte Carlo technique. In this study, however, we focus only on the scattering of the lines emitted from the shock-heated plasma. Our future study will address this issue properly.

The post-shock accretion flow is cooled by emitting bremsstrahlung X-rays and cyclotron optical/infrared radiation. Line cooling and Compton cooling are generally unimportant in most of the post-shock region and are thus neglected in this study. The velocity, density, and temperature profiles in the shock-heated region are assumed to be nonuniform, in contrast to previous studies, and are calculated using the hydrodynamic model (with bremsstrahlung and cyclotron cooling) described in Wu, Chanmugam, \& Shaviv (1994). Although resonant scattering in the postshock region can be important, we neglect this process in the simple model presented here, although resonance line trapping is expected to enhance the overall effects of Compton scattering.

We note that the ionisation structure and the line emissivities in the accretion column can be calculated directly (Wu et al. 2001). In this study, however, we simply assume that the $\mathrm{H}$-like and He-like lines are emitted from a particular stratum in the column and the $6.4 \mathrm{keV} \mathrm{Fe}$ line arises from the bottom stratum just above the white dwarf surface. A more detailed study of scattering effects with a more self-consistent model of the spatial variation in line emissivities and line-of-sight viewing angles will be presented elsewhere (Z. Kuncic et al., in preparation). The upstream pre-shock region is assumed to be filled with cold $\left(k T_{\mathrm{e}} \ll 1 \mathrm{keV}\right)$ plasma accreting at the local free-fall speed
$v_{\mathrm{ff}}=\left[G M_{\mathrm{W}} /\left(R_{\mathrm{W}}+z\right)\right]^{1 / 2}$, with densities determined by the accretion rate $\dot{m}$, i.e. $n_{\mathrm{e}}=\dot{m} /\left(\mu m_{\mathrm{H}} v_{\mathrm{ff}}\right)$.

The parameters that determine the structure of the postshock region are therefore the white dwarf mass $M_{\mathrm{w}}$, the white dwarf radius $R_{\mathrm{w}}$, the specific accretion rate $\dot{m}$, and the ratio of the efficiency of cyclotron cooling to bremsstrahlung cooling $\epsilon_{\mathrm{S}}$ (see Wu et al. 1994; Saxton, $\mathrm{Wu}, \&$ Pongracic 1997). While the white dwarf mass is a free input parameter, the white dwarf radius is obtained from the mass-radius relation given in Koester (1987).

\subsection{Monte Carlo Algorithm}

Line photons are injected in a stratum, and the propagation vectors of the photons are in random directions in the rest frame of the flow. For each photon, the initial propagation vector is Lorentz-transformed out of the rest frame of the emitter into the rest frame of the white dwarf. We then determine a (tentative) scattering point, the distance to which is determined using a non-linear rejection transport technique introduced by Stern et al. (1995), based on the concept of a virtual cross-section (see Nelson, Hirayama, \& Rogers 1985; Kawrakow \& Rogers 2001). We note that in Stern et al. (1995), a constant density plasma is considered. We construct a more general algorithm which integrates the mean free path over the spatially varying electron density (Cullen 2001a, 2001b). The Klein-Nishina formula is used in determining the scattering cross-section. At this scattering point, the momentum of the electron is drawn from an isotropic Maxwellian distribution of the local temperature. The momentum vector is then Lorentz transformed out of the rest frame of the local bulk flow to the rest frame of the white dwarf. A rejection algorithm is then used to decide whether to accept or reject the scattering event, with an acceptance probability $\sigma_{\mathrm{KN}} V_{\text {rel }} / 2 \sigma_{\mathrm{T}} c$ (Cullen $2001 \mathrm{~b}$ ), where $\sigma_{\mathrm{KN}}$ is the KleinNishina cross-section, $\sigma_{\mathrm{T}}$ is the Thomson cross-section, $c$ is the speed of light, and $V_{\text {rel }}$ is the velocity of the electron relative to the photon. For an accepted event, the energy and momentum changes are computed using the scattering algorithm described in Pozdnyakov, Sobol, \& Sunyaev (1983).

In each simulation, the photons are tagged and followed until they escape from the accretion column. If a photon impacts with the white dwarf surface after leaving the accretion column, it is treated as absorbed and is discarded. The remaining escape photons are binned to construct a spectrum. The most recent version of the Mersenne Twister random number generator (Matsumoto \& Nishimura 1998) was used in our simulations. A full description of the numerical algorithm can be found in Cullen (2001a).

\section{Results and Discussion}

We simulated electron scattering off $\mathrm{Fe} \mathrm{K} \alpha$ photons with emission line central energies 6.4, 6.675, and $6.97 \mathrm{keV}$ using a total of $10^{8}$ photons. We considered two white dwarf masses and radii: $M_{\mathrm{W}}=0.5 M_{\odot}, R_{\mathrm{W}}=9.2 \times$ $10^{8} \mathrm{~cm}$, and $M_{\mathrm{W}}=1.0 M_{\odot}, R_{\mathrm{W}}=5.5 \times 10^{8} \mathrm{~cm}$. For each 

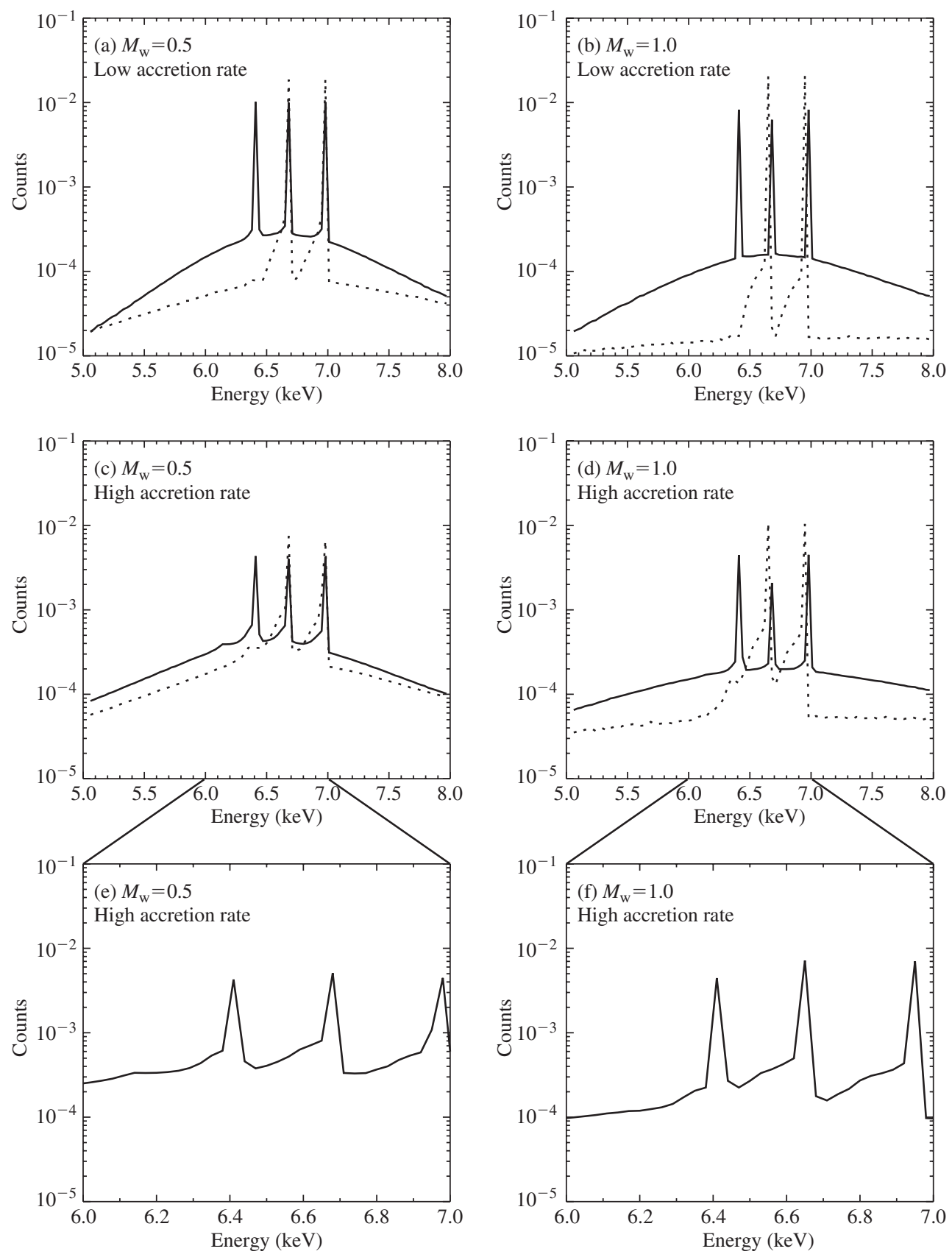

Figure 2 Simulated $\mathrm{Fe} \mathrm{K} \alpha$ emission lines scattered by electrons in the accretion column of a white dwarf of mass $0.5 M_{\odot}$ (left column) and $1.0 M_{\odot}$ (right column), and with a mass accretion rate of $1 \mathrm{~g} \mathrm{~cm}^{-2} \mathrm{~s}^{-1}$ and $10 \mathrm{~g} \mathrm{~cm}^{-2} \mathrm{~s}^{-1}$. Cyclotron cooling is set to zero. The solid and dotted lines correspond to cases for photon injection sites near the white dwarf surface and at the accretion shock, respectively. The profiles in (e) and (f) are a close-up of the $6-7 \mathrm{keV}$ region, showing the $6.4 \mathrm{keV}$ line emitted near the surface, and the 6.675 and $6.97 \mathrm{keV}$ lines emitted at the shock, under the same conditions as those for (c) and (d).

mass and radius, we considered two different mass accretion rates: $\dot{m}=1 \mathrm{~g} \mathrm{~cm}^{-2} \mathrm{~s}^{-1}$ and $\dot{m}=10 \mathrm{~g} \mathrm{~cm}^{-2} \mathrm{~s}^{-1}$. The $6.4 \mathrm{keV}$ photons were injected near the white dwarf surface, where the bulk speed of the accreting material goes to zero. The 6.675 and $6.97 \mathrm{keV}$ photons were each injected at either the white dwarf surface or the shock, where the bulk speed is equal to $v_{\mathrm{ff}} / 4$.

Figure 2 shows the results for when cyclotron cooling is negligible and bremsstrahlung cooling dominates $\left(\epsilon_{\mathrm{s}}=0\right)$. Figure 3 shows the results for when cyclotron emission is the dominant cooling process (with $\epsilon_{\mathrm{s}}=100$ ).
(A more detailed investigation into the line emissivity profile in the accretion column, taking into account collisional ionisation, is deferred for future work.) All the line profiles in Figure 2 show broadening effects due to electron (Compton) scattering. The broadening near the base of the profiles is due to multiple scattering of photons by the hot electrons in the post-shock region. This is most evident in all cases where photons are injected at the base of the accreting column (denoted by solid line profiles in Figure 2). As line photons emitted near the white dwarf surface have further to propagate through 

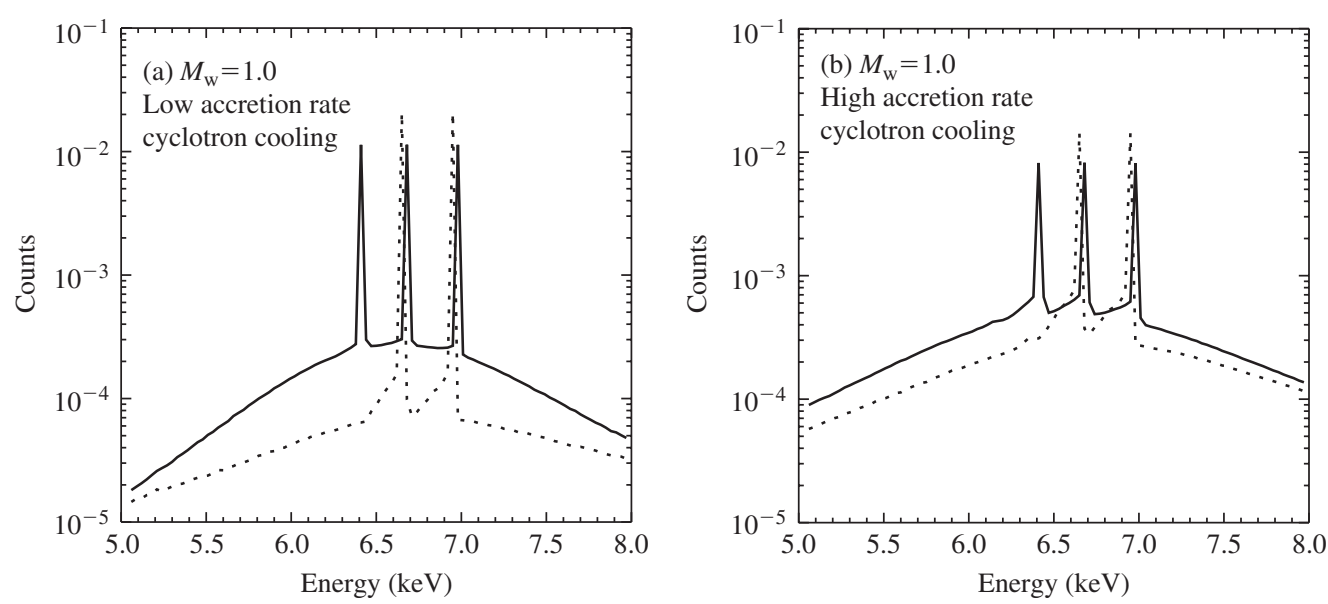

Figure 3 Simulated Fe K $\alpha$ emission lines scattered by electrons in the accretion column of a white dwarf of mass $1.0 M_{\odot}$, and mass accretion rate $10 \mathrm{~g} \mathrm{~cm}^{-2} \mathrm{~s}^{-1}$, with cooling in the post-shock region dominated by cyclotron emission. The solid and dotted lines are for photon injection sites near the white dwarf surface and at the accretion shock, respectively.

the accreting material before escaping the column, the probability of scattering is larger than that for photons emitted further above the white dwarf surface, near the shock. Line photons emitted near the shock (with profiles denoted by dotted lines in Figure 2) encounter fewer electrons in the post-shock region and thus broadening near the base of the line profiles is weaker. The broad bump caused by multiple scattering can, however, be washed out by the continuum emission (which is emitted mostly at the dense base, see Wu 2000; Cropper, Wu, \& Ramsay 2000), which also undergoes the same degree of scattering.

Distortions due to Compton recoil by cold electrons can be seen more clearly in the line profiles of photons emitted at the shock. The signature of Compton recoil, a small shoulder red-ward of the line, can be seen especially in the high- $\dot{m}$ cases, Figures $2(\mathrm{c})$ and $2(\mathrm{~d})$. This is because the electron number density increases linearly with $\dot{m}$, so the optical depth, and hence the scattering probability, increases. A comparison of Figures 2(c) and 2(d) also indicates that the shoulder-like Compton recoil signature is more conspicuous in high-mass $\mathrm{mCVs}$, if other parameters are kept constant in the model. The effects of Compton recoil scattering by cold pre-shock electrons are less likely to be washed out by continuum emission provided that the seed line photons are injected near the shock (not the dense base of the post-shock region), and that the specific accretion rate $\dot{m}$ is sufficiently high, as indicated in Figures 2(d) and 2(f).

Our simulations show that the down-scattered photons of the $\mathrm{H}$ - and He-like Fe lines emitted near the accretion shock can contaminate the neutral $\mathrm{Fe} \mathrm{K} \alpha$ line emitted near the white dwarf surface. This can be seen in Figures 2(e) and 2(f), which show close-ups of the line profiles over the energy range 6-7 keV, with the $6.4 \mathrm{keV}$ line emitted at the white dwarf surface, and the 6.675 and $6.97 \mathrm{keV}$ lines both emitted at the shock. The white dwarf mass, radius, and accretion rate are the same as in Figures 2(c) and 2(d), as indicated.
Figure 3 shows simulated line profiles with the same input parameters as those for Figures 2(b) and 2(c), but with the ratio of cyclotron to bremsstrahlung cooling $\left(\epsilon_{\mathrm{S}}\right)$ set to 100 . Qualitatively, we expect additional cooling of the electrons in the post-shock region to substantially change the thickness and the density structure of the postshock region and, therefore, the scattering optical depth. Moreover, it also alters the spatial $\mathrm{Fe} \mathrm{K} \alpha$ line emissivity profiles in the region. Our simulations in Figure 3 indicate that this enhances the overall Compton scattering effects, particularly in high $\dot{m} \mathrm{mCVs}$. Note, however, that the enhanced broadening near the base of the line profiles makes the recoil signatures less discernable. On the other hand, this also indicates that the Compton broadening of the base may be less likely than in the case of no cyclotron cooling to be washed out by continuum emissions, and thus is potentially more observable. There is some tentative evidence for this effect in Chandra High-Energy Transmission Grating (HETG) spectrum of the polar AM Her, particularly when compared to the HETG spectrum of AO Psc, which is an IP and thus has less cyclotron cooling (Hellier \& Mukai 2004). However, higher signal-to-noise observations are required to confirm this Compton scattering effect.

We note that in some mCV X-ray observations, the Fe lines appear to have centre energies slightly lower than expected (Ishida 1991; see also Hellier et al. 1998; Hellier \& Mukai 2004). In a typical observation a small red shoulder caused by recoil scattering of line photons would not be easily resolved by instruments with insufficient spectral resolution. The resulting lines in the observed spectrum would thus appear to be slightly redshifted. Thus, recoil scattering is a plausible interpretation of the observed redshifts of $\mathrm{Fe}$ lines in $\mathrm{mCV}$ X-ray spectra. Another possible interpretation, suggested by Hellier \& Mukai (2004), is that satellite lines associated with the H-like and He-like transitions are responsible for the apparent line centre shifts. These interpretations will be verifiable only when spectra with sufficiently high spectral resolution and 
signal-to-noise ratio to resolve the fine substructures in the line profiles have been obtained (e.g. by Astro-E2 and other future X-ray satellites such as Xeus and Con-X).

\section{Summary and Conclusions}

We performed Monte Carlo simulations of electron (Compton) scattering of $\mathrm{Fe} \mathrm{K} \alpha$ lines in the accretion column of mCVs using a non-linear algorithm which takes into account velocity, density, and temperature variations in the scattering region. Our results can be summarised as follows: (1) Recoil scattering by electrons in the pre-shock accretion flow can create a small shoulder-like feature redward of the line. This effect is prominent for photons emitted near the shock, but is not obvious for photons emitted from the dense base near the white dwarf surface. Moreover, the effect is most noticeable for systems with a high-mass $\left(\sim 1 M_{\odot}\right)$ white dwarf, and with a high mass accretion rate $\left(\sim 10 \mathrm{~g} \mathrm{~cm}^{-2} \mathrm{~s}^{-1}\right)$. (2) The $6.4 \mathrm{keV}$ neutral Fe line can be contaminated by the photons downscattered from the higher energy $\mathrm{H}$ - and He-like lines. (3) Lines emitted from the dense base of the accretion column experience multiple scattering, and a very broad bump is formed at the base of the line profiles. However, the broad bump is unlikely to be distinguishable from the continuum emission, which is predominantly emitted from the dense base of the column and which consequently suffers a similar degree of scattering. The possible exception to this is when cyclotron cooling is sufficiently strong to enhance the density structure in the accretion column, thereby enhancing the effects of Compton scattering in the post-shock region, and the prominence of the resulting broadening at the base of the line profiles.

\section{Acknowledgments}

Z.K. thanks the Australian Academy of Science for financial support, and the Mullard Space Science Laboratory for their hospitality. The authors also thank an anonymous referee whose comments helped to improve the paper.

\section{References}

Cropper, M. 1990, SSRv, 54, 195

Cropper, M., Wu, K., \& Ramsay, G. 2000, NewAR, 44, 57

Cropper, M., Wu, K., Ramsay, G., \& Kocabiyik, A. 1999, MNRAS, 306,684

Cullen, J. G. 2001a, PhD Thesis, University of Sydney

Cullen, J. G. 2001b, JCoPh, 173, 175

Fujimoto, R. 1998, PhD Thesis, University of Tokyo

Hellier, C., Mukai, K., \& Osborne, J. P. 1998, MNRAS, 297, 526

Hellier, C., \& Mukai, K. 2004, MNRAS, 352, 1037

Hua, X. 1997, JCoPh, 11, 660

Ishida, M. 1991, PhD Thesis, University of Tokyo

Kawrakow, I., \& Rogers, D. W. O. 2001, Proc. Monte Carlo 2000 Conf., eds. A. Kling, et al. (Berlin Heidelberg: SpringerVerlag), 135

Koester, D. 1987, ApJ, 322, 852

Lamb, D. Q., \& Masters, A. R. 1979, ApJ, 234, L117

Matsumoto, M., \& Nishimura, T. 1998, ACM Trans. on Modelling and Computer Simulation, 8(1), 3

Matt, G. 2004, A\&A, 423, 495

Nelson, W. R., Hirayama, H., \& Rogers, D. W. O. 1985, SLAC Report 265, Standford Linear Accelerator Center

Pozdnyakov, L. A., Sobol, I. M., \& Sunyaev, R. A. 1977, SvA, 21, 708

Pozdnyakov, L. A., Sobol, I. M., \& Sunyaev, R. A. 1983, ASPRv, 2, 189

Rybicki, G. B., \& Lightman, A. R. 1979, Radiative Processes in Astrophysics (New York: Wiley)

Stern, B., Begelman, M., Sikora, M., \& Svensson, R. 1995, MNRAS, 272, 291

Saxton, C. J., Wu, K., \& Pongracic, H. 1997, PASA, 14, 164

Terada, Y., et al. 2001, MNRAS, 328, 112

Warner, B. 1995, Cataclysmic Variable Stars (Cambridge: Cambridge University Press)

Wu, K. 1999, in ASP Conf. Ser. 157, Annapolis Workshop on Magnetic Cataclysmic Variables, eds. C. Hellier, \& K. Mukai (San Francisco: ASP), 325

Wu, K. 2000, SSRv, 93, 611

Wu, K., Chanmugam, G., \& Shaviv, G. 1994, ApJ, 426, 664

Wu, K., Cropper, M., \& Ramsay, G. 2001, MNRAS, 327, 208 